\title{
HISTORICAL DEVELOPMENT OF MODERN LOGIC
}

\author{
JEAN VAN HEIJENOORT
}

Modern logic began in 1879, the year in which Gottlob Frege (18481925) published his Begriffsschrift. In less than ninety pages this booklet presented a number of discoveries that changed the face of logic. The central achievement of the work is the theory of quantification; but this could not be obtained till the traditional decomposition of the proposition into subject and predicate had been replaced by its analysis into function and argument(s). A preliminary accomplishment was the propositional calculus, with a truth-functional definition of the connectives, including the conditional. Of cardinal importance was the realization that, if circularity is to be avoided, logical derivations are to be formal, that is, have to proceed according to rules that are devoid of any intuitive logical force but simply refer to the typographical form of the expressions; thus the notion of formal system made its appearance. The rules of quantification theory, as we know them today, were then introduced. The last part of the book belongs to the foundations of mathematics, rather than to logic, and presents a logical definition of the notion of mathematical sequence.

Frege's contribution marks one of the sharpest breaks that ever occurred in the development of a science. The interest in logic, so alive in the Middle Ages, had died out at the end of the fifteenth century, and the great thinkers of the next three centuries were not attracted by logical studies. In 1787 Kant wrote that logic 'is to all appearance complete and perfect'. 1 One great philosopher, Leibniz (1646-1716), showed a profound and constant interest in logic. But Leibniz's investigations are more a program than a realization. He never took the decisive steps towards a theory of quantification: that of abandoning the Aristotelian subject-

1 The reference is to the "Preface" of the second edition of Kant's Kritik der reinen Vernunft, p. viii, as quoted by W. Kneale and M. Kneale, The Development of Logic (Oxford, Clarendon Press, 1962), p. 355. 


\section{Modern Logic $\omega$}

predicate analysis of the proposition and that of taking relations seriously. After Leibniz logical studies very slowly gathered some momentum, but it is only in the middle of the nineteenth century that some decisive progress was made. George Boole (1815-1864) proposed a calculus that brought out the algebraic analogies between propositions and classes. But the fact that one and the same calculus could be interpreted both as a calculus of propositions and as a calculus of classes turned out to be a momentary drawback, rather than an asset, for the development of logic. Augustus De Morgan (1806-1871) investigated some combinatorial properties of relations, but not in a direction that would lead him to quantification theory.

So Frege's Begriffsschrift stands as a major achievement in the history of logic. Its only flaw is some confusion about quantification over functions. Frege reluctantly accepted such quantification because it is needed in his logical definition of sequence, hence of natural number (when the 'ancestral' of a relation is introduced). ${ }^{2}$ If no special safeguards are prescribed, quantification over functions opens the door to a paradox, as Bertrand Russell (1872-1970) had to inform Frege on 16 June 1902 (see van Heijenoort 1967, pp. 124-128).

Russell's paradox, that of the class of classes that do not contain themselves as an element, was only one of the paradoxes that made their appearance at the turn of the century; among the most important were the paradox of the greatest ordinal (Burali-Forti, 1897), that of the set of not finitely definable natural numbers (Richard, 1905) or real numbers (König, 1905). The paradoxes were extremely disconcerting to those then engaged in investigating the logical foundations of mathematics, and, together with other factors, they led to four major new developments, which occurred within a very short span of time:

(1) Hilbert's metamathematics (1904);

(2) Brouwer's intuitionism (1907);

(3) Zermelo's axiomatic set theory (1908);

(4) Russell's theory of types (1908).

2 Van Heijenoort has encircled the word 'reluctantly' and written a question mark in the left-hand margin. 
Hilbert's metamathematics was, at that time, a rudimentary and vague program, which was to be developed only twenty years later. Brouwer's intuitionism was a profound new conception of mathematics that demanded much time to be developed and understood. Axiomatic set theory, as presented by Zermelo, had then little import for logic, because Zermelo showed little concern for logic as a science, even when it had direct bearing upon his axiomatization (axiom of separation).

So in the early twentieth century Russell's theory of types turned out to be of major importance for the development of logic. It apparently made logic safe from paradoxes, it had flexibility and power, and it could express large tracts of mathematics, both their statements (theorems) and their arguments (proofs). It was incorporated in Principia mathematica, published by Russell and Whitehead in three volumes (1910-1913).

The logic that had then come out of Frege's and Russell's investigations is distinguished by a number of specific features. Unlike Boole and De Morgan, Frege did not consider that the task of logic is to investigate, with the use of ordinary language, combinatorial relations between propositions, but rather that it is to set up a reconstructed language, free from ambiguity and vagueness, in which mathematics (and subsequently perhaps other sciences) could be translated and developed. This new language becomes the logician's language. It is not a mere manipulation of signs, because it is, like any language, understood (according to certain rules). But it is self-sufficient: it is not interpreted in another system, such as naive set theory for instance. Since any doctrine (set theory like any other) ultimately has to be logically grounded, for Frege it would have been absurd to consider a set-theoretic semantic as a standard by which to gauge the completeness of his system. ${ }^{3}$

One consequence of this 'universal' or 'absolute' aspect of Frege's logic is that, in his system, the quantifiers range over all objects. Boole's and De Morgan's 'universes of discourse' can be changed at will; they are mathematical devices, and they have no ontological import. For Frege the ontological furniture of the universe divides into objects and functions

\footnotetext{
3 Van Heijenoort added " $a$ " in "consider a set-theoretic semantic" by hand, then encircled the " $a$ " and underlined "set-theoretic semantic". In the left-hand margin, a question mark and two verticle bars mark this passage.
} 


\section{Modern Logic $\omega$}

(concepts are functions of a special kind), and the quantifiers range over the fixed universe of objects and functions. ${ }^{4}$ When quantifiers have to be applied to a special class of objects ('for every natural number - - -', 'there exists a natural number such that ...'), Frege uses a device that is in fact the relativization of quantifiers ('for every object $x$, if $x$ is a natural number, then - - -", 'there exists an object $x$ such that $x$ is a natural number and ...'). When a function is introduced, it has to be defined for all objects (addition, for example, is defined, not only for natural numbers, but also for, say, 1 and the Moon; what the value of the function is is, in that case, irrelevant, but it must exist). With the theory of types, Russell's quantifiers cannot range over all objects; but they range over all objects of a given type. And here again, though stratified, the universe is fixed.

In 1915 Leopold Löwenheim (1878-1957) published a paper that attracted little attention at the time but turned out to be of major importance for the development of logic. Here are Löwenheim's main contributions to logic in this paper.

(1) For the first time first-order logic is taken as a distinct object of study; all questions of quantification over predicates or functions are pushed aside;

(2) Löwenheim methodically exploits the semantic approach to logic; he has no axioms or rules of inference; he uses the notion of set-theoretic interpretation and deals with questions of validity (satisfiability) in various domains;

(3) A very disquieting theorem is proved: if a formula of the firstorder predicate calculus with identity is satisfiable, it is satisfiable in a finite or a denumerably infinite domain; the theorem introduces a divorce between logic and Cantorian set theory; logic cannot deal with the nondenumerable, or rather with the distinction between denumerable and nondenumerable; the theorem is an unexpected result of modern logic that will have profound consequences;

(4) The solution of important cases of the decision and reduction problems: the singulary first-order logic is shown to be decidable, and full

\footnotetext{
4 The notation in the left-hand margin indicates that " $\&$ functions" should be added after "objects".
} 
first-order logic is reduced to binary first-order logic (with a small number of predicate letters).

Löwenheim's paper contains another notable contribution to logic, namely the method he uses to prove his theorem. This method was subsequently exploited and developed by Skolem, Herbrand, and Gödel. It constitutes the very core of Herbrand's approach to logic, and we will examine it below.

Löwenheim's set-theoretic semantic renewed an early tradition in logic, that of Peirce and Schröder. Frege's and Russell's approach to logic had been syntactic, in the sense that their systems are based on axioms and rules of inference. Moreover, this logic is, unlike Löwenheim's, formal. But here we have to remark that the word 'formal' can be used in two quite different senses:

(1) It refers to typographically verifiable properties of formulas; it is the sense that the word has in 'formal system', and it is in that sense that the Frege-Russell kind of logic is formal (although there are in Russell some relapses from that kind of formalism);

(2) An Aristotelian syllogism is formal because its validity is independent of the meaning of the terms used ('men', 'mortals', 'Athenians'); the syllogism would remain valid if these terms were replaced by other terms of the same grammatical form (class nouns or adjectives); if we adopt an extensional point of view, to each term there will correspond a set (and to relational terms there will correspond a set of ordered pairs, ordered triples, and so on). So the combination of this interpretation of the word 'formal' and the extensional viewpoint leads to the set-theoretic approach to logic.

In this (semantic) approach the basic notion is that of a valid formula, while in the syntactic approach it is that of a provable formula. The latter approach was that of Frege and Russell; the first, that of Löwenheim. At this point the question arises of the relationship between these two approaches. That every provable formula is valid constitutes the (easily solved) problem of soundness. That every valid formula is provable is the (much deeper) problem of completeness. Frege and Russell entirely ignored the set-theoretic approach; nevertheless the first-order part of their systems (that is, leaving out quantification over functions or predicates) is complete. This is undoubtedly attributable to their logical good sense, 


\section{Modern Logic $\omega$}

because, if they had carried out, as 'scratch-work', any completeness proof, they could not have failed to hit upon the Löwenheim-Skolem theorem (which is a by-product of such a proof).

By reintroducing in logic the set-theoretic semantic approach, Löwenheim opened up a new branch of logic, the theory of models, that is, the systematic investigation of the relations between syntactic objects (formulas) and set-theoretic structures. This new branch flourished several decades later, mostly under the influence of Alfred Tarski (1902-1983).

In the 1920's Thoralf Skolem (1887-1963) generalized Löwenheim's theorem, and improved its demonstration; he saw that Löwenheim's method was in fact a proof procedure, and he used it for solving new cases of the decision problem. David Hilbert (1862-1943), motivated by his investigations into the foundations of mathematics, put back in the limelight the notion of formal system.

In his doctoral dissertation (1930, written in 1929) Jacques Herbrand (1908-1931) presented a theorem that revealed a profound feature of firstorder logic. Herbrand's theorem states that a first-order formula $F$ is provable if and only if a certain quantifier-free formula, obtained from $F$ through an effective procedure, it is sententially valid. But the theorem deserves a more precise presentation. Let $Q$ be some standard first-order predicate calculus whose sentential connectives are negation and disjunction. Let $F$ be a formula of $Q$ in which no variable has bound and free occurrences and in which no two quantifiers bind the same variable (given any formula of $Q$, there is an equivalent, and easily found, formula satisfying these conditions). Let a variable be restricted in $F$ if it is bound by an existential quantifier occurring in the scopes of an even number of negation signs or by a universal quantifier occurring in the scopes of an odd number of negation signs. Let $\Gamma=\{\underline{0}, \underline{1}, \underline{2}, \ldots\}$ be an infinite list of variables of $Q$ not occurring in $F$.

By way of illustration, assume that the restricted variables of $F$ are $x_{1}$ and $x_{2}$, that its nonrestricted variables are $y_{1}, y_{2}$, and $y_{3}, y_{1}$ being free, the quantifier binding $y_{2}$ being in the scopes of the quantifiers binding $x_{1}$ and $x_{2}$, and the quantifier binding $y_{3}$ being in the scope of the quantifier binding $x_{2}$ (we shall speak of $x_{1}$ and $x_{2}$ as the 'arguments' of $y_{2}$, of $x_{2}$ as the 'argument' of $y_{3}$ ). We write the following table. 


$\begin{array}{cccccc}\text { Line } & x_{1} & x_{2} & y_{1} & y_{2}\left(x_{1}, x_{2}\right) & y_{3}\left(x_{2}\right) \\ 1 & \underline{0} & \underline{0} & \underline{1} & \underline{2} & \underline{3} \\ 2 & \underline{0} & \underline{1} & \underline{1} & \underline{4} & \underline{5} \\ 3 & \underline{1} & \underline{0} & \underline{1} & \underline{6} & \underline{3} \\ 4 & \underline{1} & \underline{1} & \underline{1} & \underline{7} & \underline{5} \\ 5 & \underline{0} & \underline{2} & \underline{1} & \underline{8} & \underline{9}\end{array}$

The table is written according to the following rules:

(1) Under the restricted variables we shall write elements of $\Gamma$ occurring on the previous lines of the table, except for the first line, on which we write $\underline{\underline{0}}$ under each restricted variable; on a given line these elements form an ordered pair, and the order in which these ordered pairs are written is determined by the following rules: if $\max (i, j)<\max (k, l)$, $\langle i, j\rangle$ precedes $\langle k, l>$; if $\max (i, j)=\max (k, l)$, the relative order of $\langle i, j\rangle$ and $\langle k, l\rangle$ is their lexicographic order.

(2) On a given line, the element of $\Gamma$ written under an unrestricted variable is different from any element of $\Gamma$ previously written in the table (on a line above or on the same line but to the left). except in the following case: if a line $q$ the 'arguments' of the nonrestricted variable $u$ have been assigned the same symbols as on line $p$, with $p<q$, then under $u$ on line $q$ we write the symbol occurring under $u$ on line $p$ (if the nonrestricted variable $v$ has no 'argument', then on every line the same symbol is written under $v$ ).

If the reader writes a few additional lines in the table above, he will grasp the method quickly.

Each line of the table determines a substitution instance of $F$ : in $F$ we delete all quantifiers and replace each variable by the symbol assigned to it in the line. Let $A_{k}$, with $k=1,2,3, \ldots$, be the substitution instance obtained by the use of line $k$. The formula

$$
A_{1} \vee A_{2} \vee \ldots \vee A_{k}
$$

which we will denote by $D_{k}$, is called the $k$ th Herbrand disjunction of $F$. Note that $D_{k}$ is a quantifier-free formula of $Q$.

Herbrand's theorem can now be stated: 


\section{$\$$ Modern Logic $\omega$}

$F$ is provable in $Q$ if and only if for some $k$ the $k$ th Herbrand disjunction of $F$ is sententially valid.

This remarkable theorem establishes a rather unexpected bridge between quantification theory and the sentential calculus.

To verify, for some $F$ and some $k$, that the $k$ th Herbrand disjunction of $F$ is sententially valid requires no ingenuity; it is a purely mechanical operation. So the function $\phi_{\mathrm{F}}(k)$ that is equal to 0 or 1 according as, for $F$, $D_{k}$, is valid or not is effectively computable (recursive; in fact, primitive recursive), and the set of formulas of $Q$ that have a valid Herbrand disjunction is recursively enumerable.

But there are additional results.

Once we have checked that, for some $k, D_{k}$ is sententially valid, we can derive $F$ from $D_{k}$ by successively introducing quantifiers and cutting repeated disjuncts in a disjunction. To be more precise, the system $Q$ is equivalent to a system $Q_{H}$ whose axioms are the sententially valid formulas of $Q$ and whose rules are (1) a rule of universalization, which allows us to reintroduce a nonrestricted quantifier at its proper place inside $F$, (2) a rule of existentialization, which allows us to do the same for a restricted quantifier, and (3) a rule of simplification, which from $M \vee N \vee N^{\prime} \vee P$ allows us to infer $M \vee N \vee P$, where $N^{\prime}$ is an alphabetical variant of $N$, and $M$ and $P$ may be empty.

These rules are one-premiss rules, and the two-premiss rule of detachment is shown to be unnecessary for first-order logic. This means that proofs can be put in a standard, and relatively simple, form: when written in a tree form, the proof has just one branch, and this result may render metamathematical arguments about proofs simpler.

Besides being two-premiss, the rule of detachment has the further disadvantage that a formula $B$, inferred through the rule from $A$ and $A \supset$ $B$, does not yield any information about $A$. In $Q_{H}$ all formulas occurring in a proof whose first line is $D_{k}$ and last line is $F$ are subformulas of $F$, substitution instances of subformulas of $F$ ('subformulas' in the extended sense), or disjunctions of such formulas. The system $Q_{H}$ has the very valuable 'subformula property'. 
If now, for a given $F, D_{k}$ is not sententially valid, for every $k$, then, for every $k, \sim D_{k}$, which is equivalent to

$$
\sim A_{1} \& \sim A_{2} \& \ldots \& \sim A_{k}
$$

is sententially satisfiable. From the fact that for every $k$ the set $\left\{\sim A_{1}, \sim A_{2}\right.$, $\left.\ldots, \sim A_{k}\right\}$ is satisfiable in a finite domain one can infer that the infinite set $\left\{\sim A_{1}, \sim A_{2}, \ldots\right\}$ is satisfiable in a denumerably infinite domain (König's lemma, or law of infinite conjunction). And from this fact one can readily infer that the formula $\sim F$ is satisfiable in a denumerably infinite domain. Hence we can now state: Either for some $k$ the $k$ th Herbrand disjunction of $F$ is valid, and in that case $F$ is provable in $Q$ (and even in $Q_{H}$ ), or for no $k$ is the $k$ th Herbrand disjunction valid, and in that case $\sim F$ is $\aleph_{0}$-satisfiable. We thus obtain the completeness of first-order logic, combined with the Löwenheim-Skolem theorem. For this completeness is generally formulated: Every valid formula $F$ of first-order logic is provable. But this is equivalent to: $F$ is provable or $\sim F$ is satisfiable. Replacing 'satisfiable' by ' $\aleph_{0}$-satisfiable', we obtain the strengthened form: $F$ is provable or $\sim F$ is $\aleph_{0}$-satisfiable.

In inferring, as indicated above, that the infinite set $\left\{\sim A_{1}, \sim A_{2}, \ldots\right\}$ is $\aleph_{0}$-satisfiable, one applies the law of excluded middle to undecidable alternatives, and the argument is not considered to be 'finitary', in Hilbert's sense of the word. Herbrand, who had adopted Hilbert's finitism, regarded such an argument as unacceptable and did not draw the inference, although he was aware of its possibility. In 1930 Kurt Gödel (1906-1978) published a proof of the completeness of first-order logic that follows the lines indicated above.

The Herbrand approach to logic, which consists in considering the successive Herbrand disjunctions of a formula, has two aspects, syntactic and semantic. If, as we did above, we obtain the disjunction by substituting syntactic objects, namely the variables of $Q$ that are in $\Gamma$, the Herbrand method can be considered to be a proof procedure. We have seen above that the set of formulas of $Q$ that have a valid Herbrand disjunction is recursively enumerable; hence, given a formula $F$, searching for a number $k$ such that $D_{k}$ is valid is exactly of the same complexity as searching for a proof of $F$ in any given standard system of quantification theory. And the 
soundness-and-completeness result for that proof procedure would be that a formula is valid if and only if some Herbrand disjunction of the formula is valid. We can, however, adopt another point of view. We can now consider the elements of $\Gamma$ to be, not variables of $Q$, but names of objects in a certain domain. The Herbrand disjunctions are no longer formulas of $Q$; they are expressions in a new language (compare validity in a finite domain: finitely many names are introduced, universal quantifiers are turned into conjunctions, existential quantifiers into disjunctions, then truth tables are used; we can look at the whole operation either as syntactic or as semantic). The validity, for some $k$, of the $k$ th Herbrand disjunction is regarded as a constructive substitute for set-theoretic validity, and the Herbrand theorem becomes a finitistic analogue of the soundness-andcompleteness theorem of quantification theory: a formula is, in that sense, 'constructively valid' if and only if it is provable.

In 1934 Gerhard Gentzen (1909-1943) ${ }^{5}$ introduced several systems, adequate either for the classical or for the intuitionistic first-order predicate calculus, that presented novel features, different from those of the systems then currently used (Frege, Russell, Hilbert-Ackermann, Heyting). One feature of Gentzen's systems is that they deal with sequents, that is, arrays consisting of two finite sequences of formulas separated by ' $\rightarrow$ ':

$$
A_{1}, A_{2}, \ldots, A_{m} \rightarrow B_{1}, B_{2}, \ldots, B_{n}
$$

This sequent can be read as

$$
\text { 'A } A_{1} \& A_{2} \& \text {...\& } A_{m} \text { ' entails ' } B_{1} \vee B_{2} \vee \ldots \vee B_{n} \text { '. }
$$

Another feature of these systems is that they employ relatively many rules and just one axiom (or, rather, axiom schema), namely $A \rightarrow A$. But the decisive trait is that the function of the rules, except for the cut rule, which is the analogue of the rule of detachment, and the relatively innocuous

5 Other sources give the date of Gentzen's death as 4 August 1945; see, e.g. p. viii of the Biographical sketch by Szabo in M.E. Szabo (editor), The collected papers of Gerhard Gentzen (Amsterdam/London, North-Holland, 1969; reprinted 1970), vii-viii. 
structural rules, is to allow the introduction of a connective or a quantifier in either the first or the second sequence of a sequent. Gentzen showed that the cut rule is eliminable. We thus obtain 'cut-free' proofs that proceed by a gradual construction of the formula to be proved. These proofs have the subformula property, just like proofs in Herbrand's $Q_{H}$.

For classical first-order logic Gentzen also showed that any proof can be divided into two parts: first, sentential rules establish, from axioms, a sententially valid Mittelsequenz, then the successive introduction of quantifiers leads to the formula to be proved. Thus Gentzen's Mittelsequenz corresponds to Herbrand's valid disjunction $D_{k}$. Hence Gentzen's results, at least for classical logic, bear a strong relation to Herbrand's.

Gentzen himself considered his method to be syntactic. He had rules different from those of Frege and Russell, but of essentially the same nature. Gentzen's rules, however, can be viewed from a semantic point of view. If a rule allows us to infer, from the derivability of $A$, the derivability of $B$, the same rule, read backward, allows us to infer, from the existence of a falsifying interpretation of $B$, the existence of a falsifying interpretation of $A$. Thus Gentzen's syntactic rules have a close relationship to the semantic rules that we would obtain by asking the question: if a formula $F$ is $f$ under a certain interpretation, what are the truth values of the subformulas of $F$ ? For example, if $F$ is $G \& H$, then $G$ is $f$ or $H$ is $f$, if $F$ is $G \vee H$, then $G$ is $f$ and $H$ is $f$, if $F$ is $(x) G x$, then, for some individual constant $a, G a$ is $f$, and so on. This method was codified by E.W. Beth (1908-1964): one writes in the left column of a semantic tableau the subformulas of $F$ that are $t$ in any interpretation in which $F$ is $f$ and in the right column of the tableau the subformulas of $F$ that are $f$ in any interpretation in which $F$ is $f$ (hence one starts with $F$ in the right column, the left column being empty). If the tableau is closed, that is, if the same formula occurs in the left and in the right columns, any interpretation in which $F$ is $f$ has to satisfy the impossible requirement that it makes one and the same formula both $t$ and $f$, hence there is no such interpretation, which means that $F$ is valid. The complete picture is somewhat more complicated than what this brief description can convey: when there is a disjunction in the left column or a conjunction in the right column, a tableau splits into two subtableaux; if a tableau is not closed, it may proceed indefinitely; and so on. The basic idea, however, is quite simple and leads to a remarkably 


\section{$\$$ Modern Logic $\omega$}

elegant proof procedure. This procedure can be further simplified if we remark that the left and right columns of a semantic tableau form two isomorphic trees: if a formula is in one column, its negation can be written in the other; hence one column can be suppressed, and we are led to the tree method (codified in a textbook by Richard Jeffrey, 1967), which is a very convenient proof procedure for quantification theory.

The present survey had to confine itself to the mainstream of modern logic. Several aspects of logic have been left untouched, in particular the logical systems that differ from classical logic: the various modal logics, the multivalued logics, and especially intuitionistic logic. This last logic, born from considerations about the foundations of mathematics, claims to be an alternative to classical logic, at least in the domain of mathematical arguments.

Modern times, that is, the period since 1879 , have seen a renaissance of logical studies. Two satellite fields have appeared: set theory and foundations of mathematics, and there have been many reciprocal influences between these two fields and logic. The revival of logic is also partly responsible for the growing analytical tendency in contemporary philosophy. Logical investigations form today a very lively field.

\section{References}

\section{BETH, Evert Willem}

1955 Semantic entailment and formal derivability, Mededelingen der Koninklijke Nederlandse Akademie van wetenschappen, afd. letterkunde, new series, vol. 18, no. 13, N.V. Noord-Hollandsche Uitgevers Maatschappij, Amsterdam.

BROUWER, Luitzen Egbertus Jan

1907 Over de grondslagen der wiskunde, Maas and van Suchtelen, Amsterdam and Leipzig; Noordhoff, Groningen. 
FREGE, Gottlob

1879 Begriffsschrift, eine der arithmetischen nachgebildete Formelsprache des reinen Denkens, Nebert, Halle; reprinted 1964, Olms, Hildesheim; English translation in van Heijenoort 1967, 1-82.

GENTZEN, Gerhard

1934 Untersuchungen über das logische Schliessen, Mathematische Zeitschrift 39, 176-210, 405-431.

GÖDEL, Kurt

1930 Die Vollständigkeit der Axiome des logischen Funktionenkalküls, Monatshefte für Mathematik und Physik 37, 349-360; English translation in van Heijenoort 1967, 582-591.

HERBRAND, Jacques

1930 Recherches sur la théorie de la démonstration, doctoral dissertation at the University of Paris; also Prace Towarzystwa Naukowego Warszawskiego, wydział III, no. 33; reprinted in Herbrand 1968, 35-153; English translation of Chapter 5 in van Heijenoort 1967, 525-581.

1968 Écrits logiques, Presses Universitaires de France, Paris.

HILBERT, David

1904 Über die Grundlagen der Logik und der Arithmetik, Verhandlungen des Dritten Internationalen Mathematiker-Kongress in Heidelberg vom 8. bis 13. August 1904, Teubner, Leipzig, 1905, 174-185; English translation in van Heijenoort 1967, 129-138.

JEFFREY, Richard C.

1967 Formal logic: its scope and limits, McGraw-Hill, New York.

LÖWENHEIM, Leopold

1915 Über Möglichkeiten im Relativkalkül, Mathematische Annalen 76, 447-470; English translation in van Heijenoort 1967, 228-251. 


\section{Modern Logic $\omega$}

RUSSELL, Bertrand

1908 Mathematical logic as based on the theory of types, American Journal of Mathematics 30, 222-262; reprinted in van Heijenoort 1967, 150-182.

See Whitehead, Alfred North, and Bertrand Russell

SKOLEM, Thoralf

1920 Logisch-kombinatorsiche Untersuchungen über die Erfüllbarkeit oder Bewisbarkeit mathematische Sätze nebst einem Theoreme über dichte Mengen, Videnskapsselskapetsskrifter, I. Matematisk-naturvidenskabelig klasse, no. 4; English translation of $\$ 1$ in van Heijenoort 1967, 252-263.

1928 Über die mathematische Logik, Norsk Matematisk Tidsskrift 10, 125-142; English translation in van Heijenoort 1967, 508-524.

VAN HEIJENOORT, Jean

1967 From Frege to Gödel: A source book in mathematical logic, 1879-1931, Harvard University Press, Cambridge, Massachusetts.

WHITEHEAD, Alfred North, and Bertrand RUSSELL

1910 Principia mathematica, Cambridge University Press, Cambridge, England, vol. 1.

$1912-$, vol. 2.
$1913-$, vol. 3.
$1925-$, 2nd ed., vol. 1.
$1927-$, 2nd ed., vols. 2 and 3. 